\title{
Germinação de sementes de crambe em diferentes temperaturas e substratos
}

\section{Crambe seed germination under the influence of temperature and substrate}

\author{
Felipe Gustavo Pilau ${ }^{1 *}$; Lucindo Somavilla ${ }^{2}$; Rafael Battisti ${ }^{3}$; \\ Luciano Schwerz ${ }^{4}$; Stela Maris Kulczynski ${ }^{5}$
}

\begin{abstract}
Resumo
Pertencente a família das Brassicaceae, nativo da zona Mediterrânea, o crambe é uma planta rústica que surge como mais uma alternativa produtiva, com potencial para a produção de matéria-prima para biodiesel, com destaque na qualidade de seu óleo. O trabalho teve por objetivo avaliar os efeitos de diferentes temperaturas e substratos na germinação de sementes de crambe, cultivar FMS Brilhante. $\mathrm{O}$ delineamento experimental foi o inteiramente casualizado no esquema fatorial 7 × 2 (temperaturas $\mathrm{x}$ substratos) com 4 repetições de 25 sementes. As sementes foram colocadas em substratos papel Germitest ${ }^{\circledR}$ e solo argiloso + areia e submetidas ao teste de germinação sob temperaturas de $9^{\circ} \mathrm{C}$, $12^{\circ} \mathrm{C}, 15^{\circ} \mathrm{C}, 20^{\circ} \mathrm{C}, 25^{\circ} \mathrm{C}, 30^{\circ} \mathrm{C}$ e $35^{\circ} \mathrm{C}$. Foram avaliados a porcentagem e o índice de velocidade de germinação. A temperatura de $25^{\circ} \mathrm{C}$ é a ideal para a realização do teste de germinação de sementes de crambe. Temperaturas inferiores aos $12^{\circ} \mathrm{C}$ e superiores aos $30^{\circ} \mathrm{C}$ são prejudiciais ao processo germinativo. Os substratos de papel Germitest ${ }^{\circledR}$ e solo argiloso + areia são adequados para realização do teste de germinação, desde que observado a temperatura de execução. $\mathrm{O}$ uso de substrato papel sob temperaturas menores a $20^{\circ} \mathrm{C}$ subestimam o potencial germinativo das sementes.
\end{abstract}

Palavras-chave: Crambe abyssinica, índice de velocidade de germinação, efeito térmico, germitest $^{\circledR}$

\begin{abstract}
Crambe, native from Mediterranean zone, belonging to the Brassicaceae family, is a rustic crop that emerges as a productive alternative, with a potential for the production of feedstock for biodiesel, with emphasis on its oil quality. This study aimed to evaluate the effects of temperature and substrate on seed germination crambe, FMS Brilhante cultivar. The experimental design was completely randomized in a factorial $2 \times 7$ (temperature $\mathrm{x}$ substrate) with 4 replicates of 25 seeds. The seeds were placed on substrates Germitest ${ }^{\circledR}$ paper and clay soil + sand and subjected to temperatures of $9^{\circ} \mathrm{C}, 12^{\circ} \mathrm{C}, 15^{\circ} \mathrm{C}$, $20^{\circ} \mathrm{C}, 25^{\circ} \mathrm{C}, 30^{\circ} \mathrm{C}$ and $35^{\circ} \mathrm{C}$. Seed germination percentage and germination speed index were evaluated. The temperature of $25^{\circ} \mathrm{C}$ is ideal for testing the seed germination of crambe. Temperatures below of $12^{\circ} \mathrm{C}$ and above of $30^{\circ} \mathrm{C}$ are detrimental to the germination process. The substrates germitest ${ }^{\circledR}$ paper and sand + clay soil are suitable for testing germination, since observed temperature of execution. The use of paper substrate at temperatures lower than $20^{\circ} \mathrm{C}$ underestimate the seed germination.
\end{abstract}

Key words: Crambe abyssinica, germination speed index, germination percentage, germitest ${ }^{\mathbb{R}}$

${ }^{1}$ Prof. do Dept ${ }^{\circ}$ de Fitotecnia, Universidade Federal de Santa Maria, UFSM. Santa Maria, RS. E-mail: fgpilau@smail.ufsm.br

${ }^{2}$ Discente em Agronomia, Centro de Educação Superior Norte-RS, CESNORS/UFSM, Frederico Westphalen, RS. E-mail: lucindosomavilla@hotmail.com

${ }^{3}$ Mestrando do Programa de Pós-Graduação em Engenharia de Sistemas Agrícolas, Escola Superior de Agricultura Luiz de Queiroz, ESALQ/USP, Piracicaba, SP. E-mail: r.battisti@hotmail.com

${ }^{4}$ Mestrando do Programa de Pós-graduação em Agronomia: Agricultura e Ambiente, CESNORS/UFSM, Frederico Westphalen, RS. E-mail: luagronomia@hotmail.com

${ }^{5}$ Prof $^{a}$ do Dept ${ }^{\circ}$. de Ciências Agronômicas e Ambientais. CESNORS/UFSM. Frederico Westphalen, RS. E-mail: stelamk@terra. com.br

*Autor para correspondência 
Introduzida recentemente no Brasil, a cultura do crambre (Crambe abyssinica) surge como uma alternativa produtiva para safra e safrinha. O grão se destaca pelo alto teor de óleo, em torno de $35 \%$, caracterizando-o como planta de potencial para a produção de matéria-prima para biodiesel, com destaque para os 50 a $60 \%$ de ácido erúcico, uma cadeia longa de ácidos graxos utilizado na produção de filmes plásticos, plastificantes, nylon, adesivos e isolamento elétrico (LAZZERI et al., 1997; BONDIOLI et al., 1998). Como resíduo da extração do óleo, a torta pode ser destinada à alimentação animal, como fonte protéica em concentração balanceada com fibras (SOUZA et al., 2009).

Originária da região do Mediterrâneo, e cultivada em algumas regiões tropicais e subtropicais, a cultura pertence à família das Brassicaceae, destaca-se por um ciclo produtivo relativamente curto, em média de 90 a 120 dias, requerendo aproximadamente 52 dias entre semeadura e florescimento (GOLZ, 1993). Tem como características a tolerância considerável a deficiência hídrica, solos e águas de irrigação salina e também resistência a baixas temperaturas, podendo tolerar de $-4^{\circ} \mathrm{C}$ a $-6^{\circ} \mathrm{C}$ por algumas horas, sem danos significativos (FOWLER, 1991; GOLZ, 1993), exaltando a possibilidade de cultivo em praticamente todas as regiões climáticas do Brasil.

Aavaliação da qualidade fisiológica das sementes, que define valores para fins de comercialização, é expressa, principalmente, pelo teste de germinação, onde cada espécie exige determinadas condições para expressar o seu máximo potencial genético. Como complemento ao teste de germinação podem ser realizados os testes de vigor, que representam atributos de qualidade fisiológica, não revelados no teste de germinação, sendo determinado sob condições de estresse ou medindo o declínio de alguma função bioquímica ou fisiológica (KRYZANOWSKI; VIEIRA; FRANÇA NETO, 1999). O vigor é utilizado principalmente para comparar lotes com percentagem de germinação muito semelhantes.
O conhecimento das condições ótimas para a germinação, principalmente da temperatura e do substrato é de fundamental importância, pois estes fatores variam entre as sementes de diferentes espécies. A temperatura afeta a porcentagem, a velocidade e a uniformidade de germinação e está relacionada com os processos bioquímicos (CARVALHO; NAKAGAWA, 2000). Segundo Marcos Filho (2005), existe uma temperatura ótima na qual se verifica uma máxima porcentagem e velocidade de germinação, acima e abaixo da qual a germinação é prejudicada.

O substrato tem grande influência no processo germinativo, pois fatores como estrutura, aeração, capacidade de retenção de água, grau de infestação de patógenos, entre outros, podem favorecer ou prejudicar a germinação das sementes. Segundo as Regras para Análise de Sementes-'RAS' (BRASIL, 2009), na escolha do substrato deve-se levar em consideração o tamanho da semente, sua exigência com relação à quantidade de água, sua sensibilidade ou não à luz e a facilidade que este oferece para a realização das contagens e avaliação das plântulas. A recomendação para a realização do teste de germinação de sementes de crambe, segundo as regras, é que as sementes podem ser colocadas sobre papel, entre papel ou entre areia.

Ruas et al. (2010), ao avaliarem a embebição de sementes de crambe, determinaram que apesar de o pericarpo ser permeável a água, sua retirada duplicou o percentual de germinação. Já com relação à temperatura, recomendações de cultivo direcionadas para o Estado de Dakota do Norte, Estados Unidos, apontam susceptibilidade à baixa temperatura na fase de emergência das plântulas (OPLINGER et al., 1991). Temperaturas ótimas na faixa de $15^{\circ} \mathrm{C}$ a $25^{\circ} \mathrm{C}$ e limitação severa à $5^{\circ} \mathrm{C}$, também foi detectado por Fowler (1991) atento a potencialidade de cultivo de crambe em regiões áridas ou semi-áridas, onde solo e água de irrigação são comumente salinos. 
Diante do interesse em empregar essa espécie em cultivos sucessivos e ampliar suas possibilidades de uso, devido ao seu potencial, é necessário buscar mais informações sobre as condições mais adequadas para que as sementes de crambe possam expressar o máximo de germinação, em condição de não salinidade. Dessa forma, este trabalho avaliou o comportamento germinativo das sementes de Crambe abyssinica sob diferentes temperaturas e substratos.

O trabalho foi realizado no Laboratório de Sementes da Universidade Federal de Santa Maria, campus de Frederico Westphalen, RS, no mês de Setembro de 2009. As sementes utilizadas foram de um lote da cultivar 'FMS Brilhante'.

Foram testados sete níveis de temperatura, mantidos constantes durante o período de germinação, com fotoperíodo de 12 horas, em dois substratos, num delineamento inteiramente casualizado no esquema fatorial 7 x 2 (temperatura x substratos), com 4 repetições de 25 sementes com pericarpo.

Os substratos testados foram o papel Germitest ${ }^{\circledR}$ umedecido com água destilada na quantidade de 2,5 vezes seu peso, e solo argiloso + areia na proporção de $1: 1$, umedecidos a $70 \%$ da capacidade de campo. Ambos os substratos foram colocados em caixas plásticas do tipo gerbox, sendo que no caso do papel usaram-se três camadas e as sementes foram dispostas sobre as mesmas e, no substrato solo argiloso + areia usou-se uma camada de $2 \mathrm{~cm}$ de espessura e as sementes enterradas a $1,0 \mathrm{~cm}$ de profundidade. As temperaturas testadas foram $9^{\circ} \mathrm{C}$, $12^{\circ} \mathrm{C}, 15^{\circ} \mathrm{C}, 20^{\circ} \mathrm{C}, 25^{\circ} \mathrm{C}, 30^{\circ} \mathrm{C}$ e $35^{\circ} \mathrm{C}$, fornecidas juntamente com o fotoperíodo por câmeras B.O.D. (Biochemical Oxigen Demand).

A porcentagem de germinação foi determinada por meio de uma única contagem aos 7 dias após a semeadura, computado-se apenas as plântulas normais, segundo as 'RAS' (BRASIL, 2009).

O índice de velocidade de germinação (IVG) foi estabelecido conjuntamente com o teste de germinação. As avaliações das plântulas foram realizadas diariamente a partir do terceiro dia após instalação do experimento. Para sementes em substrato de papel foram consideradas germinadas aquelas que apresentavam raiz primária maior que $2 \mathrm{~mm}$. Já para o substrato solo argiloso + areia foram consideradas germinadas as sementes que proporcionaram plântulas emergidas que apresentavam os cotilédones acima do substrato. No final do teste, de posse dos dados diários do número de plântulas normais calculou-se o IVG de acordo com a equação proposta por Maguire (1962).

Os resultados foram submetidos à análise de variância e as médias comparadas pelo teste de Tukey (5\%), sendo que os dados de porcentagem de germinação foram transformados na raiz quadrada de $(Y+0,5)$ para normalizar a distribuição, porém apresentados na forma original.

Conforme resultado observa-se que houve interação significativa entre substrato e temperatura, com diferenças entre os substratos e também entre as temperaturas, tanto na porcentagem de germinação (Tabela 1), quanto no IVG (Tabela 2). Analisando, porém, a porcentagem de germinação das sementes de crambe em relação à temperatura, dos diferentes substratos, observa-se que em papel Germitest ${ }^{\circledR}$ a máxima germinação se deu para o intervalo de $20^{\circ} \mathrm{C}$ a $30^{\circ} \mathrm{C}$, enquanto que em substrato solo argiloso + areia ocorreu em maior amplitude de temperatura, entre os $15^{\circ} \mathrm{C}$ e $30^{\circ} \mathrm{C}$. Considerando-se os extremos de temperatura $\left(9^{\circ} \mathrm{C}, 12^{\circ} \mathrm{C}\right.$ e $\left.35^{\circ} \mathrm{C}\right)$, verifica-se que ocorreu inibição da germinação em ambos os substratos. 
Tabela 1. Germinação (\%) de sementes de Crambe, submetidas a diferentes temperaturas e substratos. UFSM, Frederico Westphalen-RS, 2009.

\begin{tabular}{ccc}
\hline \multirow{2}{*}{ Temperatura } & \multicolumn{3}{c}{ Germinação (\%) } & Solo Argiloso + Areia \\
\cline { 2 - 4 } & Papel Germitest ${ }^{\circ}$ & $0 \mathrm{~A} \mathrm{c}$ \\
$1{ }^{\circ} \mathrm{C}$ & $0 \mathrm{~A} \mathrm{~d}$ & $37 \mathrm{~A} \mathrm{~b}$ \\
$15^{\circ} \mathrm{C}$ & $0 \mathrm{~B} \mathrm{~d}$ & $57 \mathrm{~A} \mathrm{ab}$ \\
$20^{\circ} \mathrm{C}$ & $22 \mathrm{~B} \mathrm{bc}$ & $75 \mathrm{~A} \mathrm{a}$ \\
$25^{\circ} \mathrm{C}$ & $40 \mathrm{~B} \mathrm{ab}$ & $75 \mathrm{~A} \mathrm{a}$ \\
$30^{\circ} \mathrm{C}$ & $71 \mathrm{~A} \mathrm{a}$ & $53 \mathrm{~A} \mathrm{ab}$ \\
$35^{\circ} \mathrm{C}$ & $73 \mathrm{~A} \mathrm{a}$ & $2 \mathrm{~A} \mathrm{c}$ \\
\hline & $8 \mathrm{~A} \mathrm{~cd}$ & \\
\hline Substrato $(\mathrm{S})$ & \multicolumn{3}{c}{ Valores de F $(5 \%)$} \\
Temperatura $(\mathrm{T})$ & $15,90^{*}$ \\
Interação $(\mathrm{S} \mathrm{x} \mathrm{T)}$ & $60,90^{*}$ \\
\hline $\mathrm{CV}(\%)$ & $8,10^{*}$ \\
\hline
\end{tabular}

Médias seguidas de mesma letra, maiúscula na linha e minúscula na coluna, não diferem entre si pelo teste de Tukey (5\%). *Significativo.

Fonte: Elaboração dos autores.

Tabela 2. Índice de velocidade de germinação (IVG) de sementes de Crambe, submetidas a diferentes temperaturas e substratos. UFSM, Frederico Westphalen-RS, 2009.

\begin{tabular}{|c|c|c|}
\hline \multirow{2}{*}{ Temperatura } & \multicolumn{2}{|c|}{ IVG } \\
\hline & Papel Germitest ${ }^{\circledR}$ & Solo Argiloso + Areia \\
\hline $9^{\circ} \mathrm{C}$ & $0,00 \mathrm{~B} \mathrm{c}$ & $0,93 \mathrm{~A} \mathrm{c}$ \\
\hline $12^{\circ} \mathrm{C}$ & $0,00 \mathrm{~B} \mathrm{c}$ & $2,67 \mathrm{~A} \mathrm{~b}$ \\
\hline $15^{\circ} \mathrm{C}$ & $1,01 \mathrm{~B} \mathrm{bc}$ & $2,80 \mathrm{~A} \mathrm{~b}$ \\
\hline $20^{\circ} \mathrm{C}$ & $2,42 \mathrm{~B} \mathrm{~b}$ & $3,89 \mathrm{~A} \mathrm{ab}$ \\
\hline $25^{\circ} \mathrm{C}$ & $4,41 \mathrm{~A} \mathrm{a}$ & $4,94 \mathrm{~A} \mathrm{a}$ \\
\hline $30^{\circ} \mathrm{C}$ & $4,70 \mathrm{~A} \mathrm{a}$ & $2,93 \mathrm{~B} \mathrm{~b}$ \\
\hline $35^{\circ} \mathrm{C}$ & $0,48 \mathrm{~A} \mathrm{c}$ & $0,15 \mathrm{~A} \mathrm{c}$ \\
\hline \multicolumn{3}{|c|}{ Valores de F (5\%) } \\
\hline Substrato (S) & \multicolumn{2}{|c|}{$18,88^{*}$} \\
\hline Temperatura (T) & \multicolumn{2}{|c|}{$53,70^{*}$} \\
\hline Interação (S x T) & \multicolumn{2}{|c|}{$10,16^{*}$} \\
\hline CV $(\%)$ & \multicolumn{2}{|c|}{29,08} \\
\hline
\end{tabular}

Médias seguidas de mesma letra, maiúscula na linha e minúscula na coluna, não diferem entre si pelo teste de Tukey (5\%). *Significativo.

Fonte: Elaboração dos autores.

De acordo com Marcos Filho (2005), a temperatura ótima para a maioria das espécies cultivadas situa-se entre os $20-30^{\circ} \mathrm{C}$, ficando as máximas entre 35 e $40^{\circ} \mathrm{C}$ e as mínimas geralmente inferiores a $15^{\circ} \mathrm{C}$.
Esta redução observada na germinação com a menor temperatura $\left(9^{\circ} \mathrm{C}\right)$ se deve a injúrias por resfriamento, as quais estão relacionadas a danificações no sistema de membranas (MARCOS FILHO, 2005). Para sementes de canola (Brassica napus L. var. oleifera cv. Westar), também 
Brassicaceae, Nykiforuk e Johnson-Flanagan (1999) observaram germinação em temperaturas inferiores, de $6^{\circ} \mathrm{C}$ e $10^{\circ} \mathrm{C}$. Tais pesquisadores, porém destacam a extrema lentidão do processo germinativo, demonstrando baixa porcentagem de germinação para $6^{\circ} \mathrm{C}$. Observaram ainda que temperaturas de $2^{\circ} \mathrm{C}$ e $6^{\circ} \mathrm{C}$ ocasionaram efeito deletério sobre a germinação de dois lotes de sementes, em função de retardo na mobilização de reservas. Quanto a redução de germinação pela maior temperatura $\left(35^{\circ} \mathrm{C}\right)$, Steiner et al. (2009), ao avaliar a correlação entre a temperatura e a porcentagem de germinação de sementes de rabanete, outra Brassicaceae, também relatam prejuízos frente ao extremo térmico de $35^{\circ} \mathrm{C}$.

Complementando a análise de germinação com o IVG, visto ser o mesmo lote de sementes frente às adversidades testadas do meio, este apresentou melhores respostas nas temperaturas de $25^{\circ} \mathrm{C}$ e $30^{\circ} \mathrm{C}$ em papel Germitest ${ }^{\circledR}$, e nas temperaturas de $20^{\circ} \mathrm{C}$ e $25^{\circ} \mathrm{C}$ em solo argiloso + areia. Entretanto, os maiores índices de germinação e IVG expressos pelas sementes de crambe, em ambos os substratos, ocorreram na temperatura de $25^{\circ} \mathrm{C}$, caracterizandose como a melhor temperatura para o teste de germinação de sementes de crambe. Esta temperatura está de acordo com as sugeridas pelas 'RAS' (BRASIL, 2009) para esta espécie, e coincidentes com os resultados de Nery, Carvalho e Fraga (2009), em adequação de um teste de germinação de semente de nabo, também Brassicaceae.

Avaliando-se os diferentes substratos em cada temperatura, observa-se que o solo argiloso + areia proporciona melhor desempenho fisiológico (germinação e IVG) das sementes de crambe frente às temperaturas de $12^{\circ} \mathrm{C}, 15^{\circ} \mathrm{C}$ e $20^{\circ} \mathrm{C}$. Acreditase que o contato da semente com o substrato ou mesmo solo é um dos fatores determinantes na porcentagem final de germinação e emergência de sementes. Em estudos com sementes de Stevia rebaudiana concluiu-se que quanto maior o contato da semente com o substrato, melhor são as respostas em germinação (CARNEIRO; GUEDES, 1992). Para sementes de florestais Albuquerque et al. (1998) também relataram interação significativa entre temperatura e substrato, destacando-se o uso da vermiculita como substrato, o qual permitiu maior contato com a semente, sendo recomendado para sementes de forma esférica.

Constata-se que a semente de crambe, por ser esférica, tem uma área de contato reduzida com o substrato papel Germitest ${ }^{\mathbb{R}}$, que associada à baixa fluidez da água em baixas temperaturas e presença de pericarpo na semente, dificultam sua passagem (RUAS et al., 2010), o que pode explicar a redução da eficácia deste substrato nas temperaturas de $12^{\circ} \mathrm{C}$ a $20^{\circ} \mathrm{C}$. Sendo assim, sugere-se evitar o uso da temperatura de $20^{\circ} \mathrm{C}$, proposta pelas 'RAS' (BRASIL, 2009), quando do uso de papel germitest ${ }^{\mathbb{B}}$ como substrato no teste de germinação de sementes de crambe, pois tais condições podem subestimar as reais expressões fisiológicas das sementes desta espécie.

A temperatura de $25^{\circ} \mathrm{C}$ é a ideal para a realização do teste de germinação de sementes de Crambe. Temperaturas inferiores aos $12^{\circ} \mathrm{C}$ e superiores aos $30^{\circ} \mathrm{C}$ são prejudiciais ao processo germinativo.

Os substratos de papel Germitest ${ }^{\circledR}$ e solo argiloso + areia são adequados para realização do teste de germinação, desde que observado a temperatura de execução. $\mathrm{O}$ uso de substrato papel sob temperaturas menores a $20^{\circ} \mathrm{C}$ subestimam o potencial germinativo das sementes.

\section{Agradecimentos}

Ao $\mathrm{CNPq}$ pelo financiamento do projeto e concessão de uma bolsa de iniciação científica. 


\section{Referências}

ALBUQUeRQUE, M. C.; RODRIGUES, T. J. D.; MINOHARA, L.; TEBALDI, N. D.; SILVA, L. M. M. Influência da temperatura e do substrato na germinação de sementes de saguaraji (Colubrina glandulosa perk. Rhamnaceae). Revista Brasileira de Sementes, Brasília, v. 20, n. 2, p. 108-111, nov. 1998.

BONDIOLI， P.; FOLEGATTI， L.; LAZZERI， L.; PALMIERI, S. Native Crambe abyssinica oil and its derivatives as renewable lubricants: an approach to improve its quality by chemical and biotechnological processes. Industrial Crops and Products, Amsterdam, v. 7, p. 231-238, jan. 1998.

BRASIL. Ministério da Agricultura, Pecuária e Abastecimento. Regras para análise de sementes. Ministério da Agricultura, Pecuária e Abastecimento. Secretaria de Defesa Agropecuária. Brasília, DF: Mapa/ ACS, 2009. 399 p.

CARNEIRO, J. W. P.; GUEDES, T. A. Influência do contato das sementes de Stevia (Stevia rebaudiana (Bert.) Bertoni), no substrato, avaliada pela função de Weibull. Revista Brasileira de Sementes, Brasília, v. 14, n. 1, p. 65-72, 1992.

CARVALHO, N. M.; NAKAGAWA, J. Sementes: ciência, tecnologia e produção. 4. ed. Jaboticabal: Funep, 2000. 588 p.

FOWLER, J. L. Interaction of salinity and temperature on the germination of crambe. Agronomy Journal, Madison, v. 83 , n. 1, p. 169-172, 1991.

GOLZ, T. Crambe. Fargo: North Dakota State University Extension Service, jan. 1993. (Alternative agriculture series, n. 4). Available at: <http://library.ndsu.edu/ repository/handle/10365/8032>. Accessed at: 03 mar. 2010.

KRYZANOWSKI, F. C.; VIEIRA, R. D.; FRANÇA NETO, J. B. Vigor de sementes: conceitos e testes: Londrina: ABRATES, 1999.

LAZZERI, L.; MATTEI, F. de; BUCELLI, F.; PALMIERI, S. Crambe oil: a potential new hydraulic oil and quenchant. Industrial Lubrification end Tribology, Bradford, v. 49, n. 2, p. 71-77, 1997.
MAGUIRE, J. D. Speed of germination-aid in selection and evaluation for seedlig emergence and vigor. Crop Science, Madison, v. 2, n. 1, p. 176-177, 1962.

MARCOS FILHO, J. Fisiologia de sementes de plantas cultivadas. Piracicaba: Fealq, 2005, 495 p.

NERY, M. C.; CARVALHO, M. L. M. de; FRAGA, A. C. Adequação do teste de germinação para sementes de nabo forrageiro. Revista Brasileira de Sementes, Londrina, v. 31, n. 2, p. 177-187, 2009.

NYKIFORUK, C. L.; JOHNSON-FLANAGAN, A. M. Storage reserve mobilization during low temperature germination and early seedling growth in Brassica napus. Plant Physiology and Biochemistry, Versailles, v. 37, n. 12, p. 939-947, 1999.

OPLINGER, E. S.; OELKE, E. A.; KAMINSKI, A. R.; PUTNAM, D. H.; TEYNOR, T. M.; DOLL, J. D.; KELLING, K. A.; DURGAN, B. R.; NOETZEL, D. M. Crambe: alternative field crops manual. St. Paul: University of Minnesota, 1991. Available at: <http:// www.hort.purdue.edu/newcrop/afcm/crambe.html>. Accessed at: 03 mar. 2010.

RUAS, R. A. A.; NASCIMENTO, G. B. do; BERGAMO, E. P.; DAUR JUNIOR, R. H.; ARRUDA, R. G. de. Embebição e germinação de sementes de crambe (Crambe abyssinica). Pesquisa Agropecuária Tropical, Goiânia, v. 40, n. 1, p. 61-65, 2010.

SOUZA, A. D. V.; FÁVARO, S. P.; ÍTAVO, L. C. V.; ROSCOE, R. Caracterização química de sementes e tortas de pinhão manso, nabo-forrageiro e crambe. Pesquisa Agropecuária Brasileira, Brasília, v. 44, n. 10, p. 1328-1335, 2009.

STEINER, F.; PINTO JUNIOR, A. S.; ZOZ, T.; GUIMARÃES, V. F.; DRANSKI, J. A. L.; RHEINHEIRMER, A. R. Germinação de sementes de rabanete sob temperaturas adversas. Revista Brasileira Ciências Agrárias, Recife, v. 4, n. 4, p. 430-434, 2009. 